\title{
Characterization of Silver Nanoparticles Thin Films with Various Thicknesses by AFM
}

\author{
Ling Li, Ling $\mathrm{Li}^{*}$, Weidong Chen, Jie Zheng, Linwen Wang, Yan Chen \\ College of Physics and Electronic Engineering, Sichuan Normal University, Chengdu 610101, China \\ Email: "lingli70@aliyun.com
}

Received 21 October 2015; accepted 5 January 2016; published 11 January 2016

\begin{abstract}
Ag nanoparticles (NPs) thin films with various thicknesses were prepared by thermal evaporation method. Atomic force microscopy (AFM) and UV-Vis spectrophotometer were used to study the surface morphology, growth nature and optical properties of the films. The results indicate that the growth rates of $\mathrm{Ag}$ particles in lateral and vertical direction are variable, and the dominant growth rate of Ag particles in lateral direction will convert into dominant growth rate in vertical direction as films grow thicker. The mean value and distribution of Ag NPs height related with the location and width of SPR (surface plasmonresonance) peak. Higher mean height shifts SPR peak to longer wavelengths, and smaller deviation results in narrower width of SPR peaks.
\end{abstract}

\section{Keywords}

Ag NPs Thin Films, Surface Morphology, AFM, SPR, Growth Nature

\section{Introduction}

Ag NPs, owing to their significant SPR absorption in the visible range of the electromagnetic spectrum, have attracted a lot of interest in recent years, and have been found several technological applications in Surface-Enhanced Raman spectroscopy (SERS), chemical and biological sensing, imaging, and nanoscale electronic and photonic devices [1]-[5]. Many efforts have been devoted to characterize surface morphology of various NPs films using AFM. Some researchers made AFM study on microstructures of silver films [13], Al films [14], gold films [15], ZAO films [16], besides, some researchers investigated the effects of microstructure on optical properties of different kinds of polysilicon thin films [17], and surface morphology and dynamic scaling behavior of ITO thin films by AFM [18]. All these investigations have characterized well the surface morphology; however, to date, no articles studies the growth nature (growth rate of Ag particles in lateral and vertical direction) of NPs films by AFM qualitatively.

In general, the location and width of SPR peak of Ag NPs thin films are strongly dependent on their size, size distribution, surface morphology, and surrounding dielectric medium [6]-[11]. The size of Ag NPs can be test by

${ }^{*}$ Corresponding author.

How to cite this paper: Li, L., Li, L., Chen, W.D., Zheng, J., Wang, L.W. and Chen, Y. (2016) Characterization of Silver Nanoparticles Thin Films with Various Thicknesses by AFM. Journal of Materials Science and Chemical Engineering, 4, 34-39. http://dx.doi.org/10.4236/msce.2016.41007 
transmission electron microscopy (TEM), scanning electron microscope (SEM), and atomic force microscope (AFM). Here, we used AFM combined with analysis software to characterize Ag NPs thin films. Usually the lateral resolution is far lower than the vertical resolution as the influence of the width of data points, the shape of the probe tip, and the scan rate. The vertical resolution even can reach to $0.01 \mathrm{~nm}$ with AFM [12]. Therefore, in this article we focus on analysis of vertical data of AFM testing results.

In this article, we report a study on the surface morphology and growth nature of Ag NPs films by vertical data of AFM testing results. UV-Vis spectrophotometer was used to measure the optical properties of Ag NPs thin films. The results of this letter provide a new insight into the growth rate of Ag particles in lateral and vertical direction, and the correlation of microstructure with optical properties of Ag NPs thin films with various thicknesses.

\section{Experiment}

\subsection{Fabrication of Ag NPs Thin Films}

Ag NPs thin films with various thickness (5, 7, 10, 12 and $15 \mathrm{~nm}$ ), were prepared by thermal evaporation method on polished float glass substrates in vacuum of $5 \times 10^{-4} \mathrm{~Pa}$ at room temperature. The evaporation material was Ag (99.99\%). The glasses were ultrasonically cleaned in acetone, ethanol and deionizer water for $10 \mathrm{mi}-$ nutes, respectively. Then, they were dried by nitrogen gas. At last, they were put in an oven at $120^{\circ} \mathrm{C}$ for $30 \mathrm{mi}-$ nutes to remove residual solvent. After preparing, Ag thin films with various thicknesses respectively were deposited onto the substrates. The deposition rate and thickness of Ag NPs thin films were gauged by an Inficon quartz crystal microbalance (SQC310). The deposition rate was approximately $0.05 \mathrm{~nm} \cdot \mathrm{s}^{-1}$.

\subsection{Surface Morphologies Characterization}

The surface morphology of Ag NPs thin films were studied by an AFM (Bruker Dimension Edge). The tapping mode was conducted to measure samples surface morphologies. And the scan sizes were fixed at $2 \mu \mathrm{m} * 2 \mu \mathrm{m}$ and the height of the surface relief was recorded at a resolution of 256 pixels * 256 pixels. For each sample, a variety of scans were obtained at random locations on the surface of Ag NPs thin films. In order to analysis the AFM images, all image data were converted into Nanoscope software (version 1.40.0.0). Comprehensive parameters including the root mean square $\left(R_{q}\right)$, mean particle height and its distribution, surface skewness, and particle diameter were obtained. The definition of surface skewness is as follow

$$
\text { Skewness }=\frac{1}{R_{q}^{3}} \frac{1}{N} \sum_{j=1}^{N} Z_{j}^{3}
$$

where $R_{q}$ is the $R_{m s}$ roughness. Skewness is a non dimensional quantity, which is typically evaluated in terms of positive or negative. When Skewness is negative, the valleys are dominant over the scanned area, and the peaks are dominant on the surface when Skewness is positive. When Skewness is zero, an even distribution of data around the mean data plane is suggested, and when Skewness is strongly non-zero, an asymmetric, one tailed distribution is suggested, such as a flat plane having a small, sharp spike $(>0)$, or a small, deep pit $(<0)$.

\subsection{Transmittance Spectra of Ag NP Thin Films}

The transmittance spectra of Ag NPs thin films were recorded using a UV-Vis-NIR spectrophotometer (PerkinElmer Lambda 850). The location and width of SPR peaks of the transmittance spectra correlate with the microstructure of Ag NPs thin films [19].

\section{Results and Discussion}

Figures 1(a)-(e) show three-dimensional AFM images of Ag NPs thin films with various thicknesses of 5, 7, 10, 12 and $15 \mathrm{~nm}$, respectively. The corresponding one-dimensional AFM surface profile scans are also plotted in Figure 2. One typical morphological feature is visually recognized in these Figures. The feature is that the surfaces of Ag NPs thin films show island-like structures, and these islands become larger in both lateral and vertical directions as thicker thickness. When the thickness is $5 \mathrm{~nm}$ (Figure 1(a)) and $7 \mathrm{~nm}$ (Figure 1(b)), the smaller and lower particles attached to the surface of substrate can be observed. When the thickness conti- 


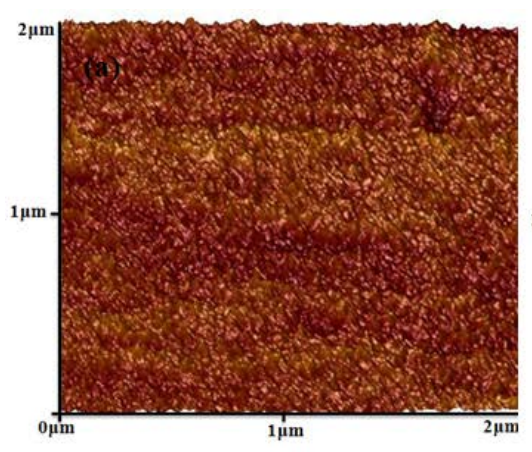

(a)

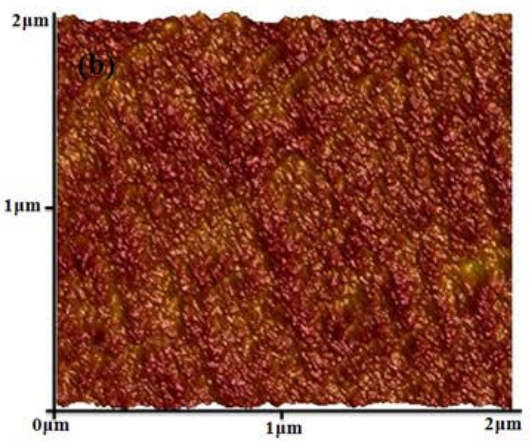

(b)

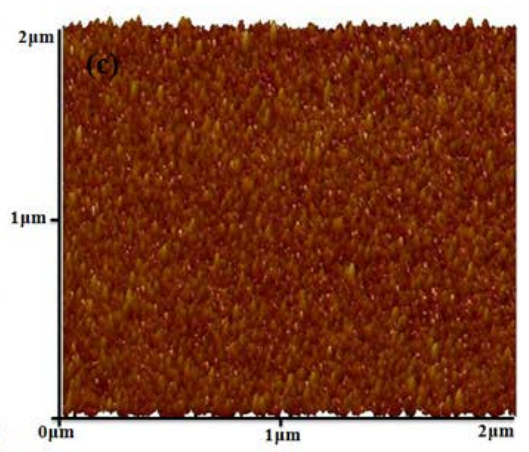

(c)

Figure 1. Three-dimensional AFM image series of Ag NPs thin films: (a) $5 \mathrm{~nm}$; (b) $7 \mathrm{~nm}$; (c) $10 \mathrm{~nm}$; (d) $12 \mathrm{~nm}$ and (e) $15 \mathrm{~nm}$.

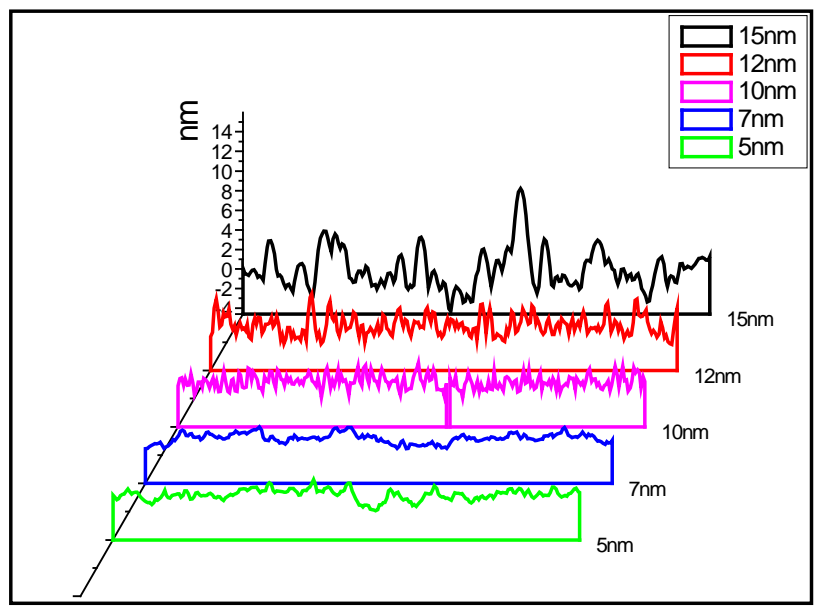

Figure 2. One-dimensional AFM surface profile scans of Ag NP films for: (a) 5 nm, (b) 7 nm, (c) 10 nm, (d) 12 nm, (e) 15 nm.

nuously increases from 10 to $15 \mathrm{~nm}$, the Ag particles grow rapidly in lateral and vertical direction forming an island-like structure, which is quite prominent when the deposition thickness is $15 \mathrm{~nm}$. So, its roughness increases from $0.714 \mathrm{~nm}$ to $1.98 \mathrm{~nm}$ as thickness increases from $10 \mathrm{~nm}$ to $15 \mathrm{~nm}$.

In Figure 2, we can see that surface fluctuation is relatively flat when the thicknesses are 5 and $7 \mathrm{~nm}$. While, the surface fluctuation becomes severely when the thickness increases from $10 \mathrm{~nm}$ to $15 \mathrm{~nm}$. So peak-to-valley values are $4.573 \mathrm{~nm}$ (5 nmAg), $4.681 \mathrm{~nm}$ (7n mAg), $6.133 \mathrm{~nm}$ (10 nmAg), $9.764 \mathrm{~nm}$ (12 nmAg), and $14.177 \mathrm{~nm}$ (15 nmAg), respectively. We can also see that from the picture the width of the peaks or valleys increases gradually from $16 \mathrm{~nm}$ (10 nmAg) to $41 \mathrm{~nm}$ (15 nmAg), which is used to estimate the average particle size.

Next, we study the symmetry of the variations of the surface topography about the mean plane by means of the parameter of surface skewness which relies on the relative height and amount of peaks and valleys. According to the Equation (1) and Figure 2, we obtain the skewness and find it increases from -0.0716 to 0.627 with the thickness increasing. To further explore the change of skewness, we made a curve about thickness and skewness in Figure 3. The skewness value increases sharply as the thickness is increased from $5 \mathrm{~nm}$ to $7 \mathrm{~nm}$, but the rate of increasing varies slowly as the thickness thicker. The results indicate that the amount of peaks sharply increases owing to the relative height of peaks and valleys almost keeping the same from Figure $\mathbf{2}$ with thickness increasing from $5 \mathrm{~nm}$ to $7 \mathrm{~nm}$. So, the amount of Ag NPs increases sharply at this stage, that is to say, the growth rate of Ag particles in lateral is dominated.

In order to statistically explore the Ag NPs height and distribution, we made histogram of the 2D surface-height values from the respective to pographies. As shown in Figure 4, the abscissa represents Ag NPs heights, and ordinate represents a percentage. Here, we can seeAg NPs height value is smaller than $4 \mathrm{~nm}$ when the thickness is $5 \mathrm{~nm}$ and $7 \mathrm{~nm}$, but that of thickness with $7 \mathrm{~nm}$ is more concentrated. However, this is not 


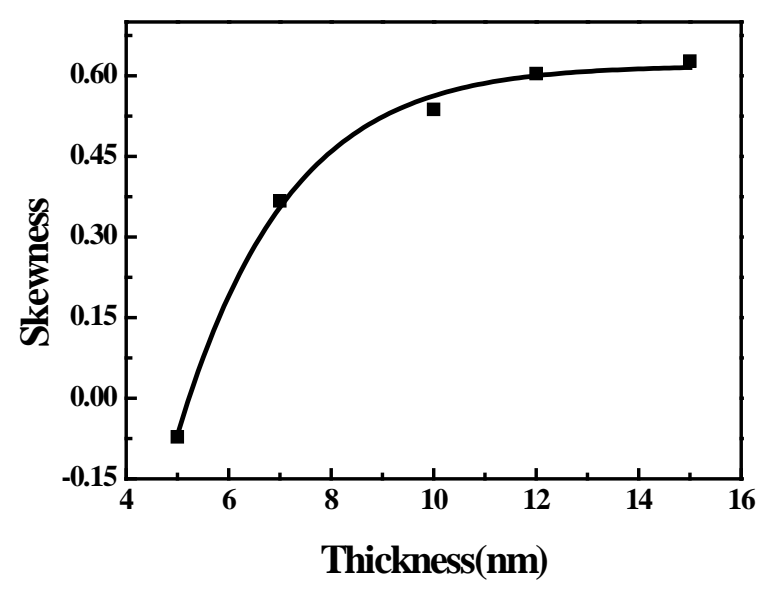

Figure 3. The curve of skewness particle height corresponding deposition thickness.

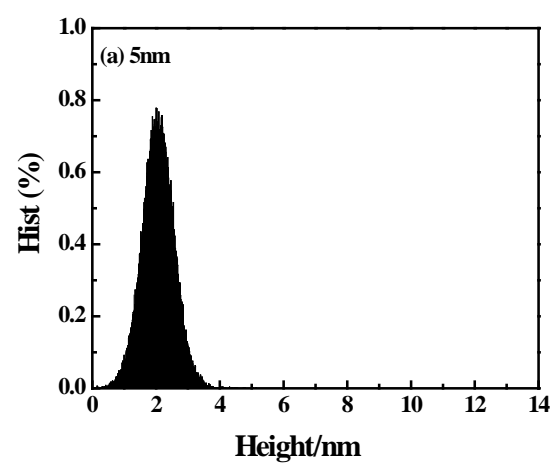

(a)

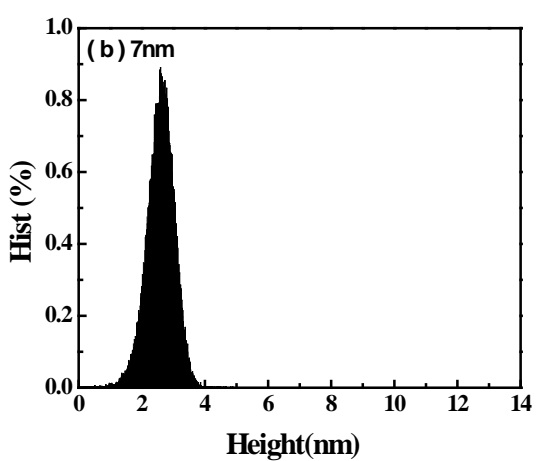

(b)

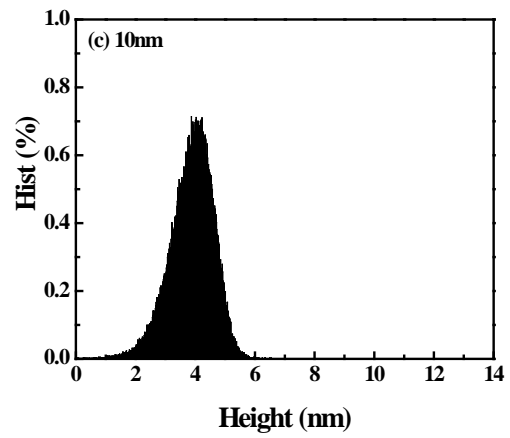

(c)

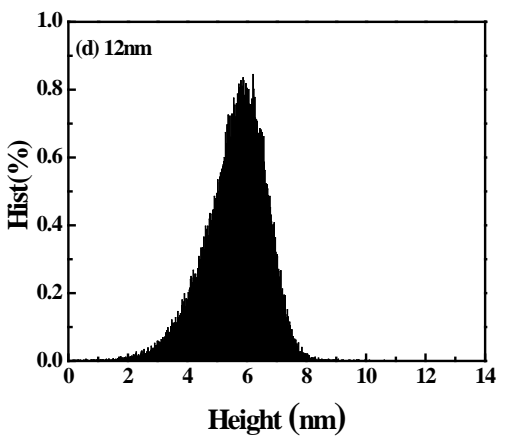

(d)

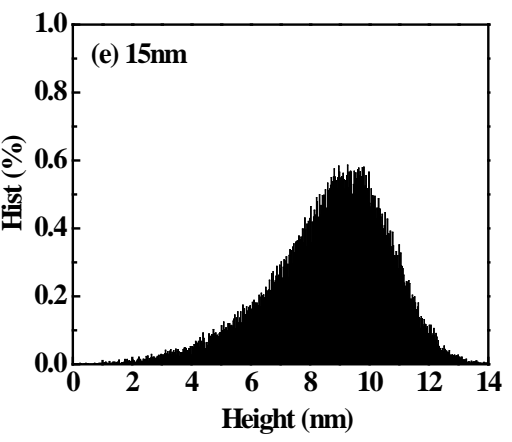

(e)

Figure 4. Histogram of the 2D surface-height values from the respective topography. (a) $5 \mathrm{~nm}$, (b) $7 \mathrm{~nm}$, (c) $10 \mathrm{~nm}$, (d) 12 $\mathrm{nm}$ and (e) $15 \mathrm{~nm}$.

clearly observed by three-dimensional AFM images and one-dimensional profile. Table 1 gives the particles mean height value with various thickness which are $2.07 \mathrm{~nm}$ (5 nm Ag), $2.6 \mathrm{~nm}$ (7 nm Ag), $3.88 \mathrm{~nm}$ (10 nm Ag), $5.61 \mathrm{~nm}(12 \mathrm{~nm} \mathrm{Ag})$, and $8.66 \mathrm{~nm}$ (15 nm Ag), respectively. These results indicate that the Ag NPs mean height value become larger as the thickness increasing.

Besides, to observe the increasingrate of mean height of AgNPs, we drew a curve about thickness and particle mean height value, as shown in Figure 5. Here we focus on the increasing rateof mean height value (the slope of curve). In the initial deposition stage, the increasing rate of mean height value is slow when thickness increases from $5 \mathrm{~nm}$ to $7 \mathrm{~nm}$, but it begins to increase quickly as the thickness thicker. Figure 5 actually reflects the change of growth rate of Ag particles in vertical direction on the substrate surface as the thickness thicker. The slope of the curve indicates that the growth rate of Ag particles in vertical direction is small when thicknesses 
increase from $5 \mathrm{~nm}$ to $7 \mathrm{~nm}$, but it gradually increases as thickness thicker. Taking the analysis about skewness into account, we can make a conclusion that the growth rate of Ag particles in lateral and vertical vary with films thickness. In the initial stage, the growth rate of Ag particles in lateral direction is dominant when thickness increases from $5 \mathrm{~nm}$ to $7 \mathrm{~nm}$ and then the growth rate of Ag particles in vertical direction is dominant when thickness increases from $12 \mathrm{~nm}$ to $15 \mathrm{~nm}$.

In this article, we not only analysis the microstructure of AgNPs thin films and their growth nature, but also explore the correlation between microstructures and optical properties. Figure 6 presents the transmittance spectra of AgNPs thin films. The locations of SPR peaks are also included in Table 1. It can be seen that the optical properties of Ag NPs thin films are related to the mean value and distribution of Ag NPs height. Higher Ag NPs correspond to longer wavelength of SPR peak. Besides, more concentrated distribution of Ag NPs result in narrower width of SPR peak.

\section{Summary}

In this article, we have analyzed the change of surface morphologies, growth rate of Ag particles in lateral and vertical direction and optical properties of Ag NPs thin films with various thickness which were fabricated by thermal evaporation deposition. AFM images and corresponding parameters (surface skewness and mean height) reveal the information that the growth rate of Ag particles in lateral and vertical is not constant. The dominant growth rate of Ag particles in lateral direction will convert into dominant growth rate in vertical direction as the films grow thicker. The mean value and distribution of Ag NPs height correlate with the location and width of

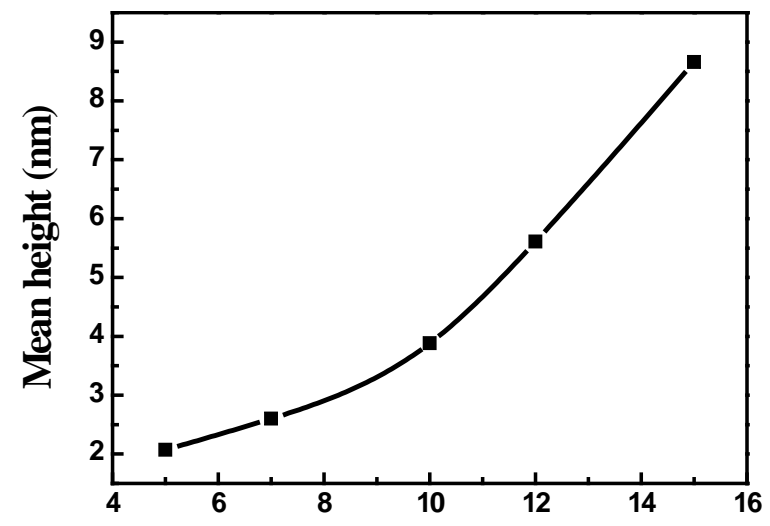

Thickness(nm)

Figure 5. The curve of mean particle height corresponding deposition thickness.

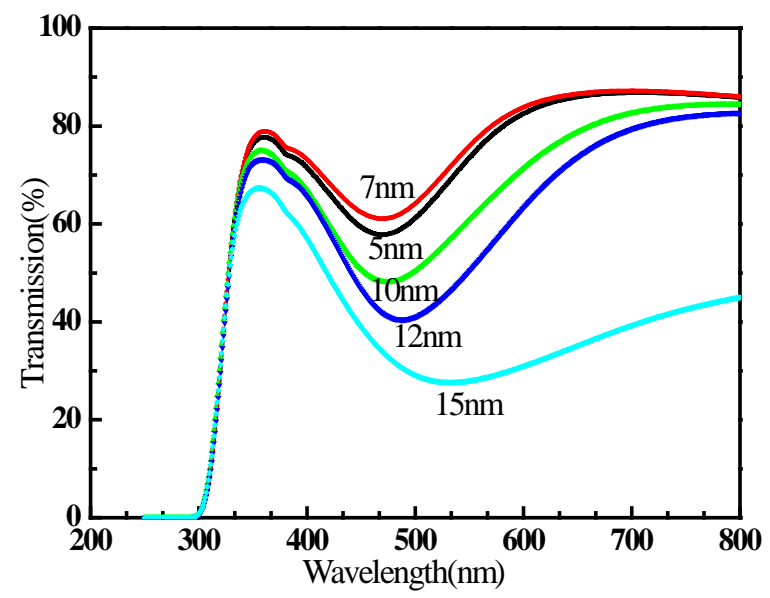

Figure 6. The transmittance spectra of Ag NPs thin films with different thickness. 
SPR peak, respectively. Higher particle heights shift SPR peak to longer wavelengths. The smaller deviation in the particle height results in narrower SPR peaks.

\section{Acknowledgements}

This work was supported by State Key Laboratory of Metastable materials Science and Technology, Yanshan University (201509), and State Key Laboratory of Optical Technologies on Nano-Fabrication and MicroEngineering, Institute of Optics and Electronics, Chinese Academy of Sciences.

\section{References}

[1] Xu, W.G., Ling, X., Xiao, J., Dresselhaus, M.S., Kong, J., Xu, H. and Zhang, J. (2012) Surface Enhanced Raman Spectroscopy on a Flat Grapheme Surface. Proceedings of the National Academy of Sciences, 109, 9281-9286. http://dx.doi.org/10.1073/pnas.1205478109

[2] Kamakshi, K., Sekhar, K.C., Almeida, A., Moreira, J.A. and Gomes, M.J.M. (2014) Tuning the Surface Plasmon Resonance and Surface-Enhanced Raman Scattering of Pulsed Laser Deposited Silver Nanoparticle Films by Ambience and Deposition Temperature. Journal of Optics, 16, 055002. http://dx.doi.org/10.1088/2040-8978/16/5/055002

[3] Haes, A.J., Stuart, D.A., Nie, S. and Van Duyne, R.P. (2004) Using Solution-Phase Nanoparticles, Surface-Confined Nanoparticle Arrays and Single Nanoparticles as Biological Sensing Platforms. Journal of Fluorescence, 14, 355-367. http://dx.doi.org/10.1023/B:JOFL.0000031817.35049.1f

[4] Fang, N., Lee, H., Sun, C. and Zhang, X. (2005) Sub-Diffraction-Limited Optical Imaging with a Silver Superlens. Science, 308, 534-537. http://dx.doi.org/10.1126/science.1108759

[5] Fang, Y., Li, Z., Huang, Y., Zhang, S., Nordlander, P., Halas, N.J. and Xu, H. (2010) Branched Silver Nanowires as Controllable Plasmon Routers. Nano Letters, 10, 1950-1954. http://dx.doi.org/10.1021/nl101168u

[6] Kreibig, U. and Vollmer, M. (1995) Optical Properties of Metal Clusters.

[7] Kelly, K.L., Coronado, E., Zhao, L.L. and Schatz, G.C. (2003) The Optical Properties of Metal Nanoparticles: The Influence of Size, Shape, and Dielectric Environment. The Journal of Physical Chemistry B, 107, 668-677. http://dx.doi.org/10.1021/jp026731y

[8] Kreibig, U. and Genzel, L. (1985) Optical Absorption of Small Metallic Particles. Surface Science, 156, 678-700. http://dx.doi.org/10.1016/0039-6028(85)90239-0

[9] Persson, B.N.J. (1991) Surface Resistivity and Vibrational Damping in Adsorbed Layers. Physical Review B, $44,3277$. http://dx.doi.org/10.1103/PhysRevB.44.3277

[10] Yang, L., Li, G.H. and Zhang, L.D. (2000) Effects of Surface Resonance State on the Plasmon Resonance Absorption of Ag Nanoparticles Embedded in Partially Oxidized Amorphous Si Matrix. Applied Physics Letters, 76, 1537-1539. http://dx.doi.org/10.1063/1.126088

[11] Peng, S., McMahon, J.M., Schatz, G.C., Gray, S.K. and Sun, Y. (2010) Reversing the Size-Dependence of Surface Plasmon Resonances. Proceedings of the National Academy of Sciences, 107, 14530-14534. http://dx.doi.org/10.1073/pnas.1007524107

[12] Jinfa, T., Peifu, G. and Xu, L. (2006) Modern Optical Thin Film Technology. Press of Zhejiang University, Hangzhou, PRC, 103.

[13] Hong, J.H., Wang, H.B., Wei, Q.F. and Gao, W.D. (2006) AFM Analysis and Conductivity of Nano-Structured Silver Film Deposited by Magnetron Sputtering. Journal of Textile Research, 27, 14.

[14] Lita, A.E. and Sanchez Jr., J.E. (1999) Characterization of Surface Structure in Sputtered Al Films: Correlation to Microstructure Evolution. Journal of applied physics, 85, 876-882. http://dx.doi.org/10.1063/1.369206

[15] Xie, J.P., Yu, W.Y., Zhang, S.L., Chen, M.G. and Ye, G.X. (2007) AFM Study on Microstructures of Metal Films Deposited on Liquid Substrates. Physics Letters A, 371, 160-164. http://dx.doi.org/10.1016/j.physleta.2007.06.015

[16] Kumar, B.R. and Rao, T.S. (2012) AFM Studies on Surface Morphology, Topography and Texture of Nanostructured Zinc Aluminum Oxide Thin Films. Digest Journal of Nanomaterials and Biostructures, 7, 1881-1889.

[17] Ren, N., Liu, Z. and Wang, Q. (2015) Investigating the Effects of Microstructure on Optical Properties of Different Kinds of Polysilicon Thin Films. Physics Letters A, 379, 1153-1160. http://dx.doi.org/10.1016/j.physleta.2015.02.023

[18] Davood, R. and Faegh, H. (2012) Surface Morphology Dynamics in ITO Thin Films. Journal of Modern Physics.

[19] Bonyar, A., Wimmer, B. and Csarnovics, I. (2013) Investigation of Thermally Generated Gold Nanoparticles with AFM. IEEE 19th International Symposium for Design and Technology in Electronic Packaging (SIITME), Galati, 2427 October 2013, 31-34. IEEE. http://dx.doi.org/10.1109/SIITME.2013.6743639 